Georg Erdmann

\title{
Comment on Clive L. Spash
}

Abstract: The basic message put forward by Spash is sound, namely that cost-benefit analysis (CBA) can hardly be used to address the question of how much money society should optimally spend in order to avoid greenhouse gas (GHG) emissions. However, the points in Spash's paper against the use of CBA for the GHG assessment vary in their significance.

Spash puts forward three arguments and I will comment on each of them. The first argument claims that some CBA studies use incorrect estimates for the benefits and costs of GHG pollutions. This may be due to input data as well as the estimation methods themselves. This argument gives no reason to reject the CBA as a tool for analyzing the GHG problem; instead, it calls for an improved and corrected application of the CBA.

The second argument is more important: ignorance and uncertainty about the damages associated with today's GHG emissions make the quantification of the benefits associated with reduced GHG emissions rather undefinable. It is obviously true that any quantifiable economic method will fail in a situation of complete lack of knowledge i.e. in a situation when even probabilistic knowledge is lacking. However, concerning GHG, the knowledge will increase in the future and therewith the likelihood for a valuable use of CBA. When CBA is not applicable today, due to missing knowledge, it is not excluded that it will be a useful decision tool in the future.

This brings me to the third argument in Spash's paper. If the preferences are lexicographically ordered and harms to future generations cannot be cancelled out by goods to future generations, there is no way to use the CBA approach for assessing GHG strategies. Here I have some difficulties: why should lexicographic preferences hold particularly for the GHG issue? As far as estimated today, GHG damages are primarily in form of future economic losses. There may be damages that should theoretically be avoided 'at all costs', but if these costs turn out to be a large fraction of income, society would find it hard to pay to avoid these damages. There is rather unlikely that society will evaluate such damages at infinite costs and order preferences lexicographically for real decision making.

There is of course the distributional aspect: The CBA approach answers to the question 'what is the most efficient GHG strategy', whereby 'efficiency' is measured in terms of the discounted sum of intergenerational welfare. However, under 
a most efficient strategy future generations will tend to be worse off than contemporary generations.

Economists know this trade-off between efficiency and justice and claim that one instrument cannot improve both aspects at the same time. Accordingly, a second instrument must be introduced in addition to CBA. Such an instrument requires the definition of property rights that protect future generations from unacceptable damages from contemporary GHG emissions. Here I fully agree with Spash.

The property right approach leads to GHG emission standards that protect the rights of future generations and restrict the action space subject to the CBA. It remains to be answered what would be a rationale for the determination of such a standard. As long as the physical and ecological mechanisms of GHG damages are not known, economics cannot contribute to this question. Furthermore, this is not a defect of the CBA itself.

But economists can discuss with their means solutions for two associated problems: how should the political procedure for the standard setting be organized in the society in order to reach the right result? And, when it becomes available, what is the cheapest way to reach the GHG standard? This last question can be answered through the cost effectiveness analysis which traditionally replaces the CBA whenever the later cannot be used as a guideline for rational decision making.

Thus, CBA cannot be applied today to the GHG problem as the degree of ecological knowledge remains insufficient, but there are no fundamental reasons why it shouldn't be applied, perhaps in addition to the property rights approach, when this knowledge becomes available. 